\title{
10 Äquivalenz der verschiedenen Gesamtheiten im thermodynamischen Limes
}

Nach (9.14) gilt im großkanonischen Zustand für die relativen Schwankungen

$$
\left(\frac{\sigma(N)}{\bar{N}}\right)^{2}=\frac{k T}{V} \kappa_{T} .
$$

Solange wir nicht in der Nähe eines kritischen Punktes sind, verschwindet $\sigma(N) / \bar{N}$ im thermodynamischen Limes wie $V^{-1 / 2}$. Diese relativen Schwankungen gehen auch am kritischen Punkt gegen null, solange sich $\kappa_{T}$ wie $V^{\sigma}$ mit $0<\sigma<1$ verhält. Dies erwartet man aufgrund verschiedener heuristischer Skalenargumente, auf die wir an dieser Stelle nicht eingehen können. Deshalb erwarten wir, dass die großkanonische und die kanonische Gesamtheit im thermodynamischen Limes äquvalent werden. In Kapitel 19 wird dies noch weiter ausgeführt werden.

Ähnliche Argumente kann man auch für die Energieschwankungen in der kanonischen Gesamtheit vorbringen. Nach Gleichung (7.6) gilt

$$
\frac{\sigma(H)}{\langle H\rangle}=\frac{1}{\langle H\rangle}\left(k T^{2} C_{V}\right)^{1 / 2} .
$$

Da sowohl $C_{V}$ als auch $\langle H\rangle$ extensive Größen sind, verschwindet auch $\sigma(H) /\langle H\rangle$ im thermodynamischen Limes, außer eventuell an der Stelle eines Phasenübergangs, wo die spezifische Wärme pro Teilchen divergieren kann. Wiederum erwartet man aber auf der Basis von heuristischen Betrachtungen, dass diese Divergenz das Verschwinden der relativen Schwankungen nicht verhindert. Dann ist die kanonische Gesamtheit äquvalent zur mikrokanonischen. (Literaturhinweise zu diesem Thema werden in Kapitel 19 gegeben.) 